\title{
Spin Liquid State around a Doped Hole in Insulating Cuprates
}

\author{
Takami Tohyamat, Yasumasa Shibata, Sadamichi Maekawa, Z.-X. Shen ${ }^{1}$, \\ Naoto NAgAOSA ${ }^{2}$ and Lance L. MilleR ${ }^{3}$ \\ Institute for Materials Research, Tohoku University, Sendai 980-8577, Japan \\ ${ }^{1}$ Department of Applied Physics and Stanford Synchrotron Radiation Laboratory, Stanford University, \\ Stanford, California 94305, USA \\ ${ }^{2}$ Department of Applied Physics, University of Tokyo, Bunkyo-ku, Tokyo 113-8656, Japan \\ ${ }^{3}$ Ames Laboratory, Iowa State University, Ames, Iowa 50011, USA
}

(Received August 16, 1999)

\begin{abstract}
The numerically exact diagonalization study on small clusters of the $t-J$ model with secondand third-neighbor hopping terms shows that a novel spin liquid state is realized around a doped hole with momentum $\mathbf{k}=(\pi, 0)$ and energy $\sim 2 J$ compared with that with $(\pi / 2, \pi / 2)$ in insulating cuprates, where the spin and charge degrees of freedom are approximately decoupled. Our finding implies that the excitations in the insulating cuprates are mapped onto the $d$-wave resonating valence bond state.
\end{abstract}

KEYWORDS: spin liquid, spin-charge separation, insulating cuprates, $t$ - $J$ model

The pairing symmetry of high- $T_{c}$ superconductors has been established to be of $d_{x^{2}-y^{2}}$ type, whose gap is maximum in the direction of $\mathbf{k}=(\pi, 0)$. Such a $d$-wave gap has been observed not only in the superconducting state but also in the normal state for the underdoped superconductors by angle-resolved photoemission spectroscopy (ARPES) experiments. 1. B . The normal-state gap is called a pseudogap. In the overdoped cuprates, the pseudogap almost disappears and a flat band with sharp spectral weight emerges around the $(\pi, 0)$ point. Understanding of the electronic states at $(\pi, 0)$ is, therefore, critical in the field of cuprate superconductivity

In the insulating cuprates, ARPES experiments 1 , have revealed the characteristic excitations below the charge transfer gap with minimum binding energy at $\mathbf{k}=(\pi / 2, \pi / 2)$ : While the spectrum near $(\pi / 2, \pi / 2)$ consists of a sharp peak, the $(\pi, 0)$ spectrum is very broad. From the numerically exact diagonalization study for a $t$ - $J$ model with second- and third-neighbor hoppings $t^{\prime}$ and $t^{\prime \prime}$, respectively, i.e., the $t-t^{\prime}-t^{\prime \prime}-J$ model, 5 it has been found that the quasi-particle (QP) weight at $(\pi, 0)$ is remarkably reduced through weakening of the antiferromagnetic (AF) spin correlation induced by $t^{\prime}$ and $t^{\prime \prime}$. The resulting broad $(\pi, 0)$ spectrum in the insulator continuously evolves into the broad one at underdoping to the sharp one at overdoping. Although the numerical results have demonstrated a reasonable agreement with ARPES data, the physical origin of the broadness of the $(\pi, 0)$ spectrum remains to be elucidated. Very recently, Ronning et al. (6) $^{2}$ pursued this issue, performing ARPES experiments on $\mathrm{Ca}_{2} \mathrm{CuO}_{2} \mathrm{Cl}_{2}$, a parent compound of high- $T_{c}$ superconductors. The experimental data show a $d$-wave-like dispersion along the $(\pi / 2, \pi / 2)$ $(\pi, 0)$ line, implying that the pseudogap is remnant of the $d$-wave gap in the insulator.

*E-mail address: tohyama@imr.tohoku.ac.jp
In this Letter, we examine the dynamics of a hole in the insulator and the spin configuration around it. With realistic values of $t^{\prime}$ and $t^{\prime \prime}$, we find a dispersion consistent with that predicted by the resopating valence bond (RVB) theory $d$ with $d$-wave pairing, 8,10 where the incoherent motion of the hole plays a crucial role. These $t^{\prime}$ and $t^{\prime \prime}$ also lead to a novel spin liquid state around a hole with $\mathbf{k}=(\pi, 0)$ and excitation energy of $\sim 2 J$, where the hole causes an antiphase in the AF spin background. Correspondingly, the spin and charge degrees of freedom are approximately decoupled there, as seen from the spin and charge correlation functions. In contrast to the $(\pi, 0)$ state, the low-energy states near $(\pi / 2, \pi / 2)$ exhibit AF behavior. The implication of the spin liquid state is discussed in connection with the $d$-wave RVB state as well as the pseudogap.

We first clarify the importance of the incoherent motion of a hole by comparing the calculations of the singlehole dispersion basedon (i) a self-consistent Born approximation (SCBA) 11, 12, 13) for the $t-t^{\prime}-t^{\prime \prime}-J$ model and (ii) a spin-density wave (SDW) mean-field approximation for a Hubbard model containing the long-range hopping terms $\left(t-t^{\prime}-t^{\prime \prime}\right.$ Hubbard). In SCBA, the self-energy of a hole is determined by taking into account the coupling to the spin background. This scheme explains consistently the reduction of the dispersion from $t$ to $J$ and the incoherent part of the spectral function extending over the energy $\sim t$. The SCBA results are in good agreement with thosegf the numerically exact diagonalization (ED) method 14) In contrast to SCBA, the SDW approximation gives only a coherent motion of a hole.

The $t-t^{\prime}-t^{\prime \prime}-J$ model is given by

$$
\begin{aligned}
H= & J \sum_{\langle i, j\rangle_{1 \mathrm{st}}} \mathbf{S}_{i} \cdot \mathbf{S}_{j}-t \sum_{\langle i, j\rangle_{1 \mathrm{st}} \sigma} c_{i \sigma}^{\dagger} c_{j \sigma} \\
& -t^{\prime} \sum_{\langle i, j\rangle_{2 \mathrm{nd}} \sigma} c_{i \sigma}^{\dagger} c_{j \sigma}-t^{\prime \prime} \sum_{\langle i, j\rangle_{3 \mathrm{rd}} \sigma} c_{i \sigma}^{\dagger} c_{j \sigma}+\text { H.c. },(1)
\end{aligned}
$$



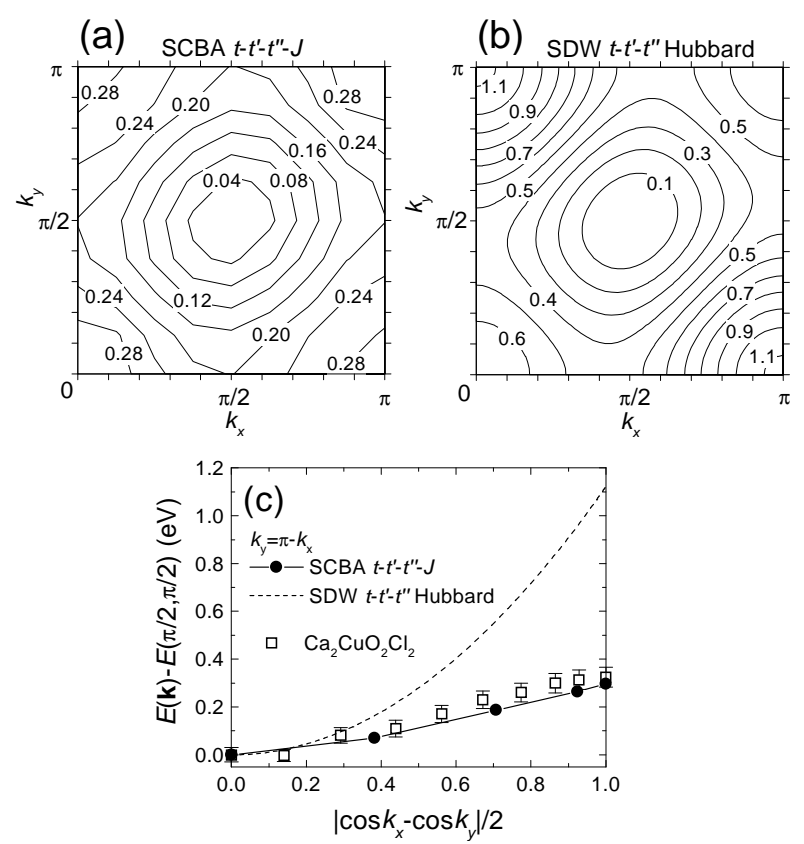

Fig. 1. (a) Contour plot of SCBA single-hole dispersion of the $t$ $t^{\prime}-t^{\prime \prime}-J$ model on a $16 \times 16$ lattice with $t=0.35 \mathrm{eV}, t^{\prime}=-0.12 \mathrm{eV}$ $t^{\prime \prime}=0.08 \mathrm{eV}$ and $J / t=0.4$. The numbers in the plot are energ. values in units of $\mathrm{eV}$ relative to the $(\pi / 2, \pi / 2)$ point. (b) $\mathrm{Th}$ same as (a) but for SDW mean-field approximation results of the half-filled $t-t^{\prime}-t^{\prime \prime}$ Hubbard model with the same hopping parameters and $U / t=10$. (c) $d$-wave plot of the dispersions along $(\pi / 2, \pi / 2)$ to $(\pi, 0)$. Dashed line: SDW result of the $t-t^{\prime}-t^{\prime \prime}$ Hubbard model. Solid circles: SCBA results of the $t-t^{\prime}-t^{\prime \prime}-J$ model. Open squares: ARPES data of $\mathrm{Ca}_{2} \mathrm{CuO}_{2} \mathrm{Cl}_{2}$ taken from ref. 6 .

where the summations $\langle i, j\rangle_{1 \text { st }},\langle i, j\rangle_{2 \text { nd }}$ and $\langle i, j\rangle_{3 \mathrm{rd}}$ run over first-, second- and third-nearest-neighbor pairs, respectively. No double occupancy is allowed, and the rest of the notation is standard. From the previous study it has been known that $t=0.35 \mathrm{eV}, t^{\prime}=-0.12 \mathrm{eV}$, and $t^{\prime \prime}=0.08 \mathrm{eV}$ for $\mathrm{Sr}_{2} \mathrm{CuO}_{2} \mathrm{Cl}_{2}$ and $\mathrm{Bi}_{2} \mathrm{Sr}_{2} \mathrm{CaCu}_{2} \mathrm{O}_{8+\delta}$. These values reveal consistent results of the Fermi surface topology with experimental data. The SDW dispersion in the AF state of the $t-t^{\prime}-t^{\prime \prime}$ Hubbard model is given by 15

$$
E_{\mathbf{k}}^{ \pm}=\varepsilon_{\mathbf{k}} \pm E_{\mathbf{k}}^{0}-\mu
$$

with

$$
\begin{aligned}
\varepsilon_{\mathbf{k}} & =-4 t^{\prime} \cos k_{x} \cos k_{y}-2 t^{\prime \prime}\left(\cos 2 k_{x}+\cos 2 k_{y}\right) \\
E_{\mathbf{k}}^{0} & =\sqrt{4 t^{2}\left(\cos k_{x}+\cos k_{y}\right)^{2}+(U m)^{2}}
\end{aligned}
$$

The chemical potential $\mu$ and the magnetization $m$ are determined by solving a set of self-consistent equations. We use the same values of $t, t^{\prime}$ and $t^{\prime \prime}$ as those for the $t-t^{\prime}-t^{\prime \prime}-J$ model, and $U / t=10$ ( $U$ is the on-site Coulomb interaction).

Figures 1(a) and 1(b) show the contour plots of the single-hole QP dispersion obtained by the SCBA for the $t-t^{\prime}-t^{\prime \prime}-J$ model on a $16 \times 16$ lattice and by the SDW approximation for the $t-t^{\prime}-t^{\prime \prime}$ Hubbard model, respectively. The SCBA contour is isotropic and thus consis-
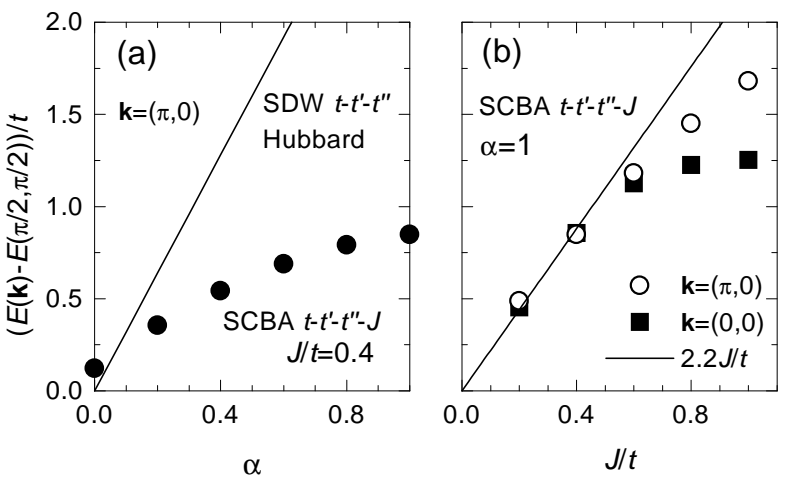

Fig. 2. (a) The dependence of the energy difference $E(\pi, 0)-$ $E(\pi / 2, \pi / 2)$ (in units of $t=0.35 \mathrm{eV}$ ) on the values of $t^{\prime}$ and $t^{\prime \prime}$. The variable $\alpha$ represents a scaling factor of $t^{\prime}$ and $t^{\prime \prime}$ as $t^{\prime}\left(t^{\prime \prime}\right)=$ $\alpha t_{0}^{\prime}\left(t_{0}^{\prime \prime}\right)$, being $t_{0}^{\prime}=-0.12 \mathrm{eV}$ and $t^{\prime \prime}=0.08 \mathrm{eV}$. Solid line: SDW result of the $t-t^{\prime}-t^{\prime \prime}$ Hubbard model given by $4 \alpha\left(2 t_{0}^{\prime \prime}-t_{0}^{\prime}\right)$. Solid circles: SCBA results of the $t-t^{\prime}-t^{\prime \prime}-J$ model on a $16 \times 16$ lattice with $J / t=0.4$. (b) $(E(\mathbf{k})-E(\pi / 2, \pi / 2))$ versus $J / t$ in the case of $\alpha=1$. The solid line represents $2.2 J / t$ known as the band width from $(\pi / 2, \pi / 2)$ to $(0,0)$.

tent with the ARPES dispersion in $\mathrm{Ca}_{2} \mathrm{CuO}_{2} \mathrm{Cl}_{2}$ that is isotropic around $(\pi / 2, \pi / 2)$, while the SDW one is not. In addition, the width of the SDW dispersion is remarkably wider than that for the SCBA results. This is clearly seen when we plot the dispersion along $(\pi / 2, \pi / 2)$ to $(\pi, 0)$. In Fig. $1(\mathrm{c})$, the energies relative to the $(\pi / 2, \pi / 2)$ point, $E(\mathbf{k})-E(\pi / 2, \pi / 2)$, are plotted against $\left|\cos k_{x}-\cos k_{y}\right| / 2$, together with the data from $\mathrm{Ca}_{2} \mathrm{CuO}_{2} \mathrm{Cl}_{2}$ which are well fitted by a straight line 6 ) What is the origin and implication of the difference between the two results? Figure 2(a) shows the dependence of the energy difference $E(\pi, 0)-E(\pi / 2, \pi / 2)$ on the values of $t^{\prime}$ and $t^{\prime \prime}$. The variable $\alpha$ represents a scaling factor of $t^{\prime}$ and $t^{\prime \prime}$ as $t^{\prime}=\alpha t_{0}^{\prime}$ and $t^{\prime \prime}=\alpha t_{0}^{\prime \prime}$, being $t_{0}^{\prime}=-0.12 \mathrm{eV}$ and $t_{0}^{\prime \prime}=0.08 \mathrm{eV}$. The SDW result is given by a function $4 \alpha\left(2 t_{0}^{\prime \prime}-t_{0}^{\prime}\right)$ [see Eq. (2)], independent of $U$. The result of the $t-t^{\prime}-t^{\prime \prime}-J$ model deviates remarkably from the SDW line and has a tendency to saturate around $\alpha \sim 1$. In addition to the slow increase of the energy with increasing $\alpha$, the wave-function renormalization $Z$ (ref. 16) at $(\pi, 0)$ is dramatically suppressed from $Z=0.87(\alpha=0)$ to $0.24(\alpha=1)$. This is again in contrast to the SDW result, where $Z$ is always one. At $\alpha \sim 1$, the energy difference is approximately $0.8 t=2 J$. This number is of crucial importance because the incoherent spectrum of a hole starts from the energy position of about $2 J$ above the spectrum of $\mathrm{QP}$ at $(\pi / 2, \pi / 2)$ (see insets in Fig. 4). Although the energy of the QP spectrum at $(\pi, 0)$ is almost the same as that at $(\pi / 2, \pi / 2)$ in the $t-J$ model, the former increases with increasing $\alpha$ and the spectrum merges into the incoherent spectra for $\alpha \sim 1$. As a result, the difference between $E(\pi, 0)$ and $E(\pi / 2, \pi / 2)$ saturates and $Z$ becomes small.

The above results indicate that when realistic values of $t^{\prime}$ and $t^{\prime \prime}(\alpha=1)$ are employed in the $t-t^{\prime}-t^{\prime \prime}-J$ model, the dispersion from $(\pi / 2, \pi / 2)$ to $(\pi, 0)$ is sensitive to the value of $J$. We now plot, in Fig. 2(b), the energy differ- 

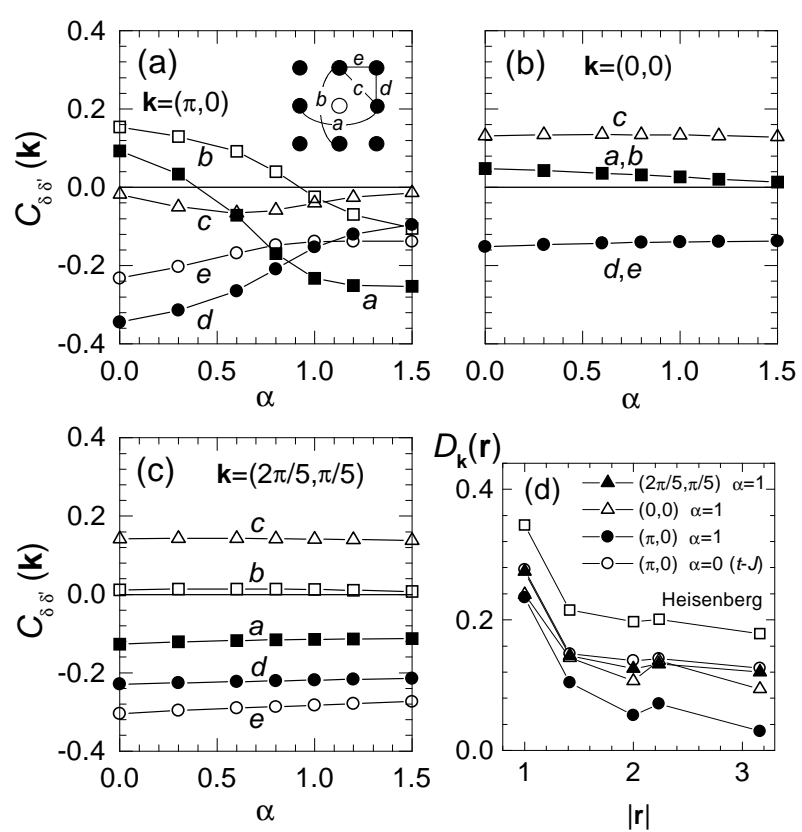

Fig. 3. (a) Spin correlation $C_{\delta \delta^{\prime}}(\mathbf{k})$ of the $t-t^{\prime}-t^{\prime \prime}-J$ model on a $2 C$ site cluster with one hole. $t=0.35 \mathrm{eV}$ and $J / t=0.4$. The variabl $\alpha$ represents a scaling factor of $t^{\prime}$ and $t^{\prime \prime}$ as $t^{\prime}\left(t^{\prime \prime}\right)=\alpha t_{0}^{\prime}\left(t_{0}^{\prime \prime}\right.$, being $t_{0}^{\prime}=-0.12 \mathrm{eV}$ and $t^{\prime \prime}=0.08 \mathrm{eV}$. $\mathbf{k}=(\pi, 0)$. (b) The same as (a) but for $\mathbf{k}=(0,0)$. (c) The same as (a) but for $\mathbf{k}=(2 \pi / 5, \pi / 5)$. The labels in (a), (b) and (c) denote configurations around a hole shown in the inset of (a). (d) The staggered spin correlation $D_{\mathbf{k}}(\mathbf{r})$ at several momenta for $\alpha=0$ and 1 of the 20 -site $t-t^{\prime}-t^{\prime \prime}-J$ cluster as a function of $|\mathbf{r}|$. The result for the Heisenberg model is also shown for comparison.

ence $E(\pi, 0)-E(\pi / 2, \pi / 2)$ as a function of $J / t$, together with the difference between $E(0,0)$ and $E(\pi / 2, \pi / 2)$ that has been known to be scaled as $\sim 2.2 J .4$ We find that $E(\pi, 0)-E(\pi / 2, \pi / 2)$ has the same $J$ dependence as $E(0,0)-E(\pi / 2, \pi / 2)$, at least, in the realistic range $J / t=0.2 \sim 0.6$. Therefore, the dispersion of the hole is governed by the spin degree of freedom. This explains why the dispersion is isotropic around $(\pi / 2, \pi / 2)$. Such an isotropic dispersion whose width is controlled by $J$ is similar to that from the flux phase or $d$-wave RVB picture. 10 .

We now examine the spin and charge characteristics of the QP state with $\mathbf{k}=(\pi, 0)$. Figure $3(\mathrm{a})$ shows the spin correlation around a hole defined as $C_{\delta, \delta^{\prime}}(\mathbf{k}) \equiv$ $\sum_{i}\left\langle\phi_{\mathrm{QP}}(\mathbf{k})\left|n_{i}^{h} \mathbf{S}_{i+\delta} \cdot \mathbf{S}_{i+\delta^{\prime}}\right| \phi_{\mathrm{QP}}(\mathbf{k})\right\rangle$, as a function of $\alpha$. Here, $\delta$ and $\delta^{\prime}$ denote two sites around the hole following the labeling convention shown in the inset. $n_{i}^{h}$ is the hole-number operator at site $i$, and $\phi_{\mathrm{QP}}(\mathbf{k})$ represents the wave function of the QP state with momentum $\mathbf{k}$. The numerically exact diagonalization method is used for a 20 -site cluster. For $\alpha=0$, i.e., the $t$ - $J$ model, the spin correlations in the $a$ and $b$ configurations [see the inset of Fig. 3(a)] are positive, i.e ferromagnetic (FM), while those for $d$ and $e$ are AF.t. With increasing $\alpha$, the correlation of $a$ changes from FM to AF and saturates near $\alpha \sim 1$. The $b$ configuration shows a similar but slow change. The configuration $d$ exhibits the opposite behavior, reducing $\mathrm{AF}$ correlation with increasing $\alpha$,

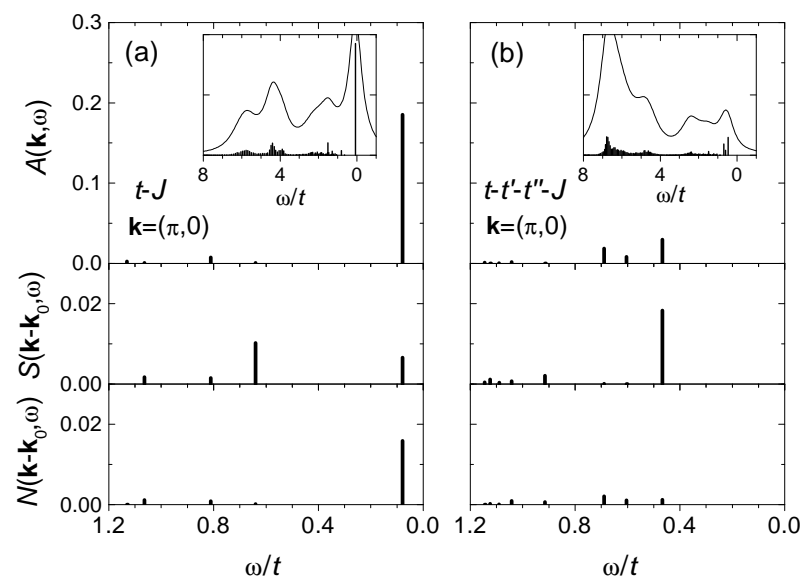

Fig. 4. $\quad A(\mathbf{k}, \omega)$ at half-filling, and $S(\mathbf{q}, \omega)$ and $N(\mathbf{q}, \omega)$ with $\mathbf{q}=\mathbf{k}-$ $\mathbf{k}_{0}$ at one-hole doping for a 20-site $t-t^{\prime}-t^{\prime \prime}-J$ model. $J / t=0.4$. $\mathbf{k}=(\pi, 0)$ and $\mathbf{k}_{0}=(3 \pi / 5,-\pi / 5)$. The energy $\omega$ in $A(\mathbf{k}, \omega)$ is measured from the QP peak at $\mathbf{k}_{0}$. (a) $t-J$ and (b) $t-t^{\prime}-t^{\prime \prime}-J$ with realistic values of $t^{\prime}$ and $t^{\prime \prime}(\alpha=1)$. The height of the bars represents the spectral intensity. The insets in (a) and (b) show $A(\mathbf{k}, \omega)$ with full-energy scale. The delta functions (vertical bars) are broadened by a Lorentzian with a width of $0.3 t$ (solid lines).

which again nearly saturates around $\alpha=1$. These behaviors from $\alpha=0$ to $\sim 1$ imply that the spin background of the $(\pi, 0)$ state changes from a Néel-like state to a spin liquid state in which spins have no tendency to order. It is interesting that the spin liquid state is accompanied by an antiphase of spins around the hole, as evidenced by AF correlation of the $a$ configuration for $\alpha \sim 1$. Since the correlation of $b$ is very small there, the antiphase of spins is one-dimensional (1D). This situation is similar to the case of a doped 1D insulator in which the spin-charge separation occurs.18) In contrast to the $(\pi, 0)$ state, QP states with different momenta do not show such remarkable changes as a function of $\alpha$, as shown in Figs. 3(b) and $3(\mathrm{c})$. We note that the AF correlation of the $a$ configuration for $\mathbf{k}=(2 \pi / 5, \pi / 5)$ is not related to the spin liquid state [see also Fig. 3(d)] but due to a twisted character gf the spin correlation seen in the one-hole ground state.17)

The staggered spin correlation $D_{\mathbf{k}}(\mathbf{r}) \equiv \sum_{i} P_{i}(\mathbf{r})$ $\left\langle\phi_{\mathrm{QP}}(\mathbf{k})\left|\mathbf{S}_{i+\mathbf{r}} \cdot \mathbf{S}_{i}\right| \phi_{\mathrm{QP}}(\mathbf{k})\right\rangle / N_{s}$ also supports the concept of the spin liquid state with $(\pi, 0)$. Here, $\mathbf{r}$ is the vector connecting two sites, $N_{s}$ is the total number of sites, and $P_{i}(\mathbf{r})= \pm 1$ depending on whether the two sites are on the same sublattice. Figure $3(\mathrm{~d})$ shows $D_{\mathbf{k}}(\mathbf{r})$ at several momenta for $\alpha=0$ and 1, together with the spin correlation of the Heisenberg model which shows AF long-range order in the thermodynamic limit. Except for the case of $\alpha=1$ at $(\pi, 0)$ (filled circles), $D_{\mathbf{k}}(\mathbf{r})$ shows $|\mathbf{r}|$ dependence similar to that of the Heisenberg model, indicating the presence of long-range order. The $(\pi, 0)$ state for $\alpha=1$, however, shows a rapid decrease of the correlation with increasing $|\mathbf{r}|$. This is again consistent with the spin liquid concept.

The dynamical properties of spin and charge degrees of freedom also provide us with useful information on the QP state with $(\pi, 0)$. We calculate the dynamical spin and charge correlation functions, $S(\mathbf{q}, \omega)$ and $N(\mathbf{q}, \omega), \underline{1})$ 
on the 20-site cluster with one hole, the Hilbert space of which is equivalent to that for the final state of the singlehole spectral function $A(\mathbf{k}, \omega)$. The momentum transfer is given by $\mathbf{q}=\mathbf{k}-\mathbf{k}_{0}$, where $\mathbf{k}_{0}$ is the ground-state momentum of the one-hole system. In Fig. $4, A(\mathbf{k}, \omega)$ with $\mathbf{k}=(\pi, 0)$ is compared with $S(\mathbf{q}, \omega)$ and $N(\mathbf{q}, \omega)$, where we take $\mathbf{k}_{0}=(3 \pi / 5,-\pi / 5)$.20 In the $t-J$ model, both spin and charge components are involved in the QP state at $\omega / t=0.08,21$ while only spin component remains in the lowest-energy state at $\omega / t=0.47$ in the $t-t^{\prime}-t^{\prime \prime}-J$ model as seen in Fig. 4(b). This is because of the separation of the spin and charge degrees of freedom. However, the separation is incomplete unlike the $1 \mathrm{D}$ case of the $t$-J model.18) We note that the results of the $t-t^{\prime}-t^{\prime \prime}-J$ model are smoothly connected to those of the $t-J$ model, showing no separation, as seen in Fig. 4(a). Much of the weight of the charge excitation shifts to a higher energy region where the spectral function has large weight $(\omega / t \sim 7)$ [see the inset of Fig. 4(b)]. For $\mathbf{k}=(2 \pi / 5, \pi / 5)$, which is close to $(\pi / 2, \pi / 2)$, the QP involves both spin and charge components independent of $\alpha$ (not shown here) 21)

We have shown that the spin background in the $(\pi, 0)$ state in the insulator behaves like a spin liquid state. At the same time, the spin and charge degrees of freedom are nearly separated in the state.22 The almost linear behavior of the dispersion near $(\pi, 0)$ in the $d$-wave plot shown in Fig. 1(c) indicates that the spin liquid state has a $d$-wave gap. All of these facts tempt us to identify the state with the $d$-wave RVB state discussed in refs. 8-10. However, we have to distinguish the present spin liquid state with the RVB state: the former is seen in the one-hole state with $\mathbf{k}=(\pi, 0)$ and the excitation energy of $\sim 2 J$, while the $d$-wave RVB theory predicts a spin liquid state as well as the spin-charge separation which are independent of the momentum of a doped hole. In fact, the present results reveal that hole states near $(\pi / 2, \pi / 2)$, i.e., the low-energy states, are not effective to destroy the AF long-range order [see Fig. 3(d)]. However, the states near $(\pi, 0)$, i.e., the high-energy $(\sim 2 J)$ states, are in good agreement with the excited states predicted by the $d$-wave RVB theory. Therefore, the spin background changes from the AF state in the low-energy-region to the spin liquid one in the high-energy region 23) The $d$ wave RVB theory might thus be regarded as an effective theory to describe the states in the high-energy region.

With hole doping, the energy of the $(\pi, 0)$ state shifts to the Fermi level, maintaining the broadness 5 that is an indication of the spin liquid state. Since the pseudogap with the order of $J$ (high-energy pseudogap) is determined by the position of the the broad spectrum, , $^{3}$ the spin liquid concept is the most probable explanation of the origin of the high-energy pseudogap in underdoped cuprates.

In summary, we have examined the dynamics of a doped hole and spin correlation around it in insulating cuprates by using the $t-t^{\prime}-t^{\prime \prime}-J$ model. In contrast to the AF state near $(\pi / 2, \pi / 2)$, we have found a novel spin liquid state around the hole with $\mathbf{k}=(\pi, 0)$ and excitation energy of $\sim 2 J$, where the spin and charge degrees of freedom are approximately decoupled. Our findings im- ply that the excitations in the insulating cuprates are mapped onto the $d$-wave RVB state.

This work was supported by the Ministry of Education, Science, Sports and Culture of Japan, CREST and NEDO. The numerical calculation was performed in the supercomputing facilities in ISSP, University of Tokyo, and IMR, Tohoku University.

[1] A. G. Loeser, Z.-X. Shen, D. S. Dessau, D. S. Marshall, C.-H. Park, P. Fournier and A. Kapitulnik: Science 273 (1996) 325.

[2] H. Ding, T. Yokoya, J. C. Campuzano, T. Takahashi, M. Randeria, M. R. Norman, T. Mochiku, K. Kadowaki and J. Giapinzakis: Nature 382 (1996) 51.

[3] D. S. Marshall, D. S. Dessau, A. G. Loeser, C.-H. Park, A. Y. Matsuura, J. N. Eckstein, I. Bozovic, P. Fournier, A. Kapitulnik, W. E. Spicer and Z.-X. Shen: Phys. Rev. Lett. 76 (1996) 4841

[4] B. O. Wells, Z.-X. Shen, A. Matsuura, D. M. King, M. A. Kastner, M. Greven and R. J. Birgeneau: Phys. Rev. Lett. 74 (1995) 964.

[5] C. Kim, P. J. White, Z.-X. Shen, T. Tohyama, Y. Shibata, S. Maekawa, B. O. Wells, Y. J. Kim, R. J. Birgeneau and M. A. Kastner: Phys. Rev. Lett. 80 (1998) 4245.

[6] F. Ronning, C. Kim, D. L. Feng, D. S. Marshall, A. G. Loeser, L. L. Miller, J. N. Eckstein, I. Bozovic and Z.-X. Shen: Science 282 (1998) 2067.

[7] P. W. Anderson: Science 235 (1987) 1996.

[8] I. Affleck and J. B. Marston: Phys. Rev. B 37 (1988) 3774.

[9] R. B. Laughlin: Phys. Rev. Lett. 79 (1997) 1726.

[10] X.-G. Wen and P.A. Lee: Phys. Rev. Lett. 76 (1996) 503.

[11] S. Schmitt-Rink, C. M. Varma, and A. E. Ruckenstein: Phys. Rev. Lett. 60 (1988) 2793.

[12] C. L. Kane, P. A. Lee and N. Read: Phys. Rev. B 39 (1989) 6880 .

[13] G. Martinez and P. Horsch: Phys. Rev. B 44 (1991) 317.

[14] T. Xiang and J. M. Wheatley: Phys. Rev. B 54 (1996) R12653.

[15] See, for example, D. Duffy and A. Moreo: Phys. Rev. B 55 (1997) R676.

[16] E. Dagotto: Rev. Mod. Phys. 66 (1994) 763.

[17] J. Inoue and S. Maekawa: J. Phys. Soc. Jpn. 59 (1990) 3467, and references therein.

[18] C. Kim, A. Y. Matsuura, Z.-X. Shen, N. Motoyama, H. Eisaki, S. Uchida, T. Tohyama and S. Maekawa: Phys. Rev. Lett. 77 (1996) 4054.

[19] T. Tohyama and S. Maekawa: J. Phys. Soc. Jpn. 65 (1996) 1902.

[20] For $\alpha \leq 0.7$, the ground state is at $( \pm \pi / 5, \pm 3 \pi / 5)$ and $( \pm 3 \pi / 5, \mp \pi / 5)$, while for $\alpha>0.7$ it is at $( \pm 2 \pi / 5, \pm \pi / 5)$ and $( \pm \pi / 5, \mp 2 \pi / 5)$. Since the energy difference between them is very small, the spin-charge separation behavior obtained does not depend on the choice of $\mathbf{k}_{0}$.

[21] S. Maekawa and T. Tohyama: Physica C 282-287 (1997) 286.

[22] Indication of the spin-charge separation around a doped hole was also discussed by G. B. Martins, R. Eder and E. Dagotto (Phys. Rev. B 60 (1999) R3716).

[23] This is consistent with the fact that the high-energy spectrum of the two-dimensional $S=1 / 2$ Heisenberg model is described in terms of the spinons of the $d$-wave RVB state. See T. C. Hsu: Phys. Rev. B 41 (1990) 11379. 